\title{
The role of focal infections in the pathogenesis of psoriasis and chronic urticaria
}

\author{
Paweł Łukasz Brzewski ${ }^{1}$, Magdalena Spałkowska ${ }^{2}$, Magdalena Podbielska², Joanna Chmielewska ${ }^{2}$, Marta Wołek $^{1}$, \\ Katarzyna Malec ${ }^{3}$, Anna Wojas-Pelc ${ }^{1}$
}

\author{
1Department of Dermatology, Jagiellonian University Medical College, Krakow, Poland \\ Head: Prof. Anna Wojas-Pelc MD, PhD \\ 2Students' Scientific Society, Jagiellonian University Medical College, Krakow, Poland \\ Head: Prof. Anna Wojas-Pelc MD, PhD \\ ${ }^{3}$ Department of Otolaryngology, Head and Neck Surgery, $5^{\text {th }}$ Military Hospital with Polyclinic, Krakow, Poland \\ Head: Andrzej Kozak MD, PhD
}

\begin{abstract}
Introduction: The Focal Infection Theory, originally presented at the beginning of the $20^{\text {th }}$ century, postulates that systemic diseases can be caused by microorganisms that arise from the focus of infection. Foci of infections have been described as sinuses, adenoids, tonsils, teeth, genitourinary tract, gall bladder and kidneys. A focus of infection is defined as the area that can occur in any part of the body, contains a pathogen (microorganism) and is usually asymptomatic. There are discordant opinions about the role of focal infections in the pathogenesis of psoriasis and urticaria.

Aim: To establish whether there is a higher incidence of focal infections in patients with chronic urticaria and psoriasis.

Material and methods: We retrospectively reviewed 129 patients with a history of psoriasis and chronic urticaria: 58 women and 71 men treated in the Department of Dermatology of the Jagiellonian University Medical College in Krakow.

Results: In the analyzed group, 11 patients had a dental consultation, 58 - laryngological consultation and 29 women had a gynecological consultation. The most common examples of focal infection were tonsillitis, upper respiratory tract infections, sinusitis, dental caries and genitourinary tract infections. Aggravating factors were similar to previously described.

Conclusions: A high incidence of focal infections in patients with psoriasis and urticaria suggests that infections may play a significant role in the pathogenesis of these skin disorders. Treatment of infection foci may play the key role in the remission of skin changes.
\end{abstract}

Key words: focal infection, urticaria, psoriasis.

\section{Introduction}

The Focal Infection Theory, originally presented at the beginning of the $20^{\text {th }}$ century, postulates that systemic diseases can be caused by microorganisms that arise from the focus of infection. Foci of infections have been described as sinuses, adenoids, tonsils, teeth, genitourinary tract, gall bladder and kidneys. A focus of infection is defined as the area that can occur in any part of the body, contains a pathogen (microorganism) and is usually asymptomatic.

\section{Chronic urticaria}

Chronic urticaria is defined by the presence of urticaria, on most days of the week, for at least 6 weeks. The diagnosis is made by description of its clinical features (typical urticarial lesions \pm angioedema) with the criteria of time. Approximately $40 \%$ of the patients with chronic urticaria have episodes of angioedema [1]. Urticaria is classified into immunological mediated (IgE-dependent and non-lgE dependent) and non-immunological mediated ones. Phys-

Address for correspondence: Paweł Łukasz Brzewski MD, PhD, Department of Dermatology, Jagiellonian University Medical College, 8 Skawińska St, 31-066 Krakow, Poland, phone: +48 12424 79 99, ext. 7400, e-mail: brzewski@gmail.com Received: 16.11.2012, accepted: 19.02.2013. 
ical urticaria is caused by physical exertion, mechanical, electromagnetic or thermal stimuli (such as water, UV light, cold, heat, pressure) with nonspecific activation of mast cells [2-4]. The most common form of chronic urticaria is the idiopathic form (80-90\%), where the external allergic cause or disease cannot be found [5-7]. There are several theories trying to explain the pathogenesis of urticaria. Chronic urticaria is usually self-limited with a duration of 2-5 years. Approximately 30-50\% of patients with chronic idiopathic urticaria have circulating auto antibodies to the high-affinity IgE receptor, Fc\&RI, on the mast cells and basophils releasing histamine or antibodies to $\lg E$ [8]. This subgroup of chronic urticaria is called autoimmune urticaria. The best in vivo clinical test for detection of in vitro basophil histamine releasing activity with $65-71 \%$ sensitivity and $78-81 \%$ specificity is the autologous serum skin test (ASST) [9]. Skin lesions in urticaria are well-circumscribed, raised above the surface of the skin, pale in the middle, with a smooth surface and extreme purities. The shape of the skin changes (plaques, wheals) varies: they can appear as round, polycyclic, annular, geographic lesions [10]. Angioedema in chronic urticaria is an episodic swelling of the mucosa or subcutaneous tissue, asymmetric, developing in a few minutes to a few hours, common in the area of the face [11]. Aggravating factors include physical factors (hot, cold, humidity, etc.), NSAIDS, stress, diet (e.g. spicy meals) [12, 13]. Many autoimmune disorders are associated with chronic urticaria. Patients with chronic urticaria have an increased odds ratio for hypothyroidism, hyperthyroidism, antithyroid antibodies. There is a significantly higher incidence of rheumatoid arthritis, Sjögren syndrome, celiac disease, type I diabetes mellitus, and systemic lupus erythematosus among female patients with chronic urticaria. All examined patients had a high mean platelet volume, positive rheumatoid factor, and antinuclear antibodies. The common mechanism in previously mentioned diseases is a high prevalence of autoantibodies and the presence of a chronic inflammatory process (high mean platelet volume). $9.8 \%$ of examined patients had hypothyroidism, $2.6 \%$ - hyperthyroidism (0.6\% and $0.5 \%$, respectively, in controls) [14]. Thyroid autoantibodies (esp. anti-TPO antibodies, antimicrosomal antibodies) are found 2-3 times more often among patients with chronic urticaria than in controls $[15,16]$. The exact role of autoantibodies remains unknown. It is suggested that patients with chronic urticaria have a higher tendency to develop autoantibodies [17]. Focal bacterial, viral, parasite infections may play the key role in autoimmunity. In the differential diagnosis of the chronic urticaria, disorders that should be taken into consideration are complement-mediated disorders, malignancies, cutaneous or systemic mastocytosis, connective tissue diseases (such as systemic lupus erythematosus, cutaneous blistering disorders (e.g. bullous pemphigoid and dermatitis herpetiformis)) [18]. The pathogenesis of chronic urticaria has not been fully described yet. The most popular theories include: the au- toimmune response, histamine releasing factors theory and cellular defects theory. Autoimmune theory claims that autoantibodies and serum factor present in blood cause release of histamine from skin mast cells and basophiles. The main argument against this theory is that antibodies found in chronic urticaria (autoantibodies to lgE, FceRI) are not specific to the disease. The other theory is supported by the fact that some patients with chronic idiopathic urticaria demonstrate excessive production of plateletderived clotting factors. This could support the theory that there are histamine releasing factors in the plasma. The cellular defects theory claims that patients with urticaria have mast cell defects (in signaling, trafficking, function) [19-23].

\section{Psoriasis}

Psoriasis is a common and chronic inflammatory skin disease with an incidence of $2.5 \%$ in Caucasians and $1.3 \%$ in African-Americans [24, 25]. Psoriasis has high potential to reduce the quality of life of the patient. Patients with psoriasis reported reduction in physical functioning and mental functioning comparable to patients with cancer, arthritis, heart disease, hypertension, diabetes and depression [26]. Psoriasis is characterized by the presence of thick, red skin with flaky, silver-white patches (scales) derived from excessive growth of skin epithelial cells. In psoriasis we distinguish the following types: plaque (psoriasis vulgaris), guttate, pustular, inverse (flexural psoriasis, intertriginous psoriasis) and erythrodermic (exfoliative psoriasis) [27]. Psoriasis is characterized by flare-ups and remissions. Main factors triggering psoriasis are: skin injuries (e.g. scratching), medications, stress, smoking, alcohol, streptococcal infections, HIV and hormonal changes [28-31]. High prevalence of obesity has been reported in patients with psoriasis [32]. In differential diagnosis of psoriasis, the following diseases should be considered: atopic keratoconjunctivitis, atopic dermatitis, adult blepharitis, dry eye syndrome, contact dermatitis, seborrheic dermatitis, diaper dermatitis, onychomycosis, squamous cell carcinoma, nummular eczema, lichen planus, lichen simplex chronicus, mycosis fungoides, subcorneal pustulosis, pustular eruptions pityriasis alba, pityriasis rosea, sicca keratoconjunctivitis, syphilis, reactive arthritis, gout and pseudogout and tinea [33]. Psoriasis is an autoimmune disease with a great role of genetic and environmental factors in its pathogenesis [34]. Factors playing a key role in the pathogenesis of psoriasis are: T cells, antigen presenting cells (APCS), keratinocytes, Langerhans' cells, macrophages, natural killer cells (NKs), an array of Th1 type cytokines, growth factors (VEGF, KGF) and others. The main hypothesis assumes that the disease starts with the activation of the $T$ cell by an unknown antigen and subsequent secretion of the cytokines by activated T cells, inflammatory cells and keratinocytes. Activated Langerhans' cells migrate to lymph nodes and present the antigen to nodal naive T cells. The $T$ cells may become antigen-specific memory cells and 
may react with keratin (cross-reaction, molecular mimicry). There are many studies supporting the role of genetic factors in the pathogenesis of psoriasis. The concordance rate for psoriasis is $63-73 \%$ in monozygotic twins, compared to $17-20 \%$ in dizygotic twins. Loci suggested as predisposing factors are PSORS1-PSORS9 [35]. The role of focal infections in the pathogenesis of psoriasis has been described for decades [36, 37]. There are single case reports of remission of skin changes after tonsillectomy [38-44].

\section{Focus of infection as the cause of systemic response}

The Focal Infection Theory, with its simplicity, has been the point of interest to scientists for decades. The first description of focal infection influencing the whole organism was the cure of the arthritis in a patient after tooth extraction, reported by Hippocrates. In 1890, doctor Miller published his dissertation "The Micro-Organisms of the Human Mouth: the Local and General Diseases Which are Caused By Them". Miller was the first scientist to use the term "focal infection" [39, 40]. 1912 is the year of actual beginning of the Focal Infection Theory. The physician Frank Billings presented the theory in the form of case reports. For the first time, he showed the role of tonsillectomy and dental extractions in the cure of systemic diseases [41, 42] The theory's golden years were the twenties, when a large number of diseases were treated with management of the focal infection [43].

A systemic response may be caused by dissemination of microorganisms from the focus. The possible role of foci in the pathogenesis of systemic diseases has been attributed to inflammatory processes such as: arthritis, neuritis, endocarditis, pancreatitis, pneumonia, asthma, Hodgkin's disease etc. More recent studies mention among diseases caused by microorganisms from focus of infection: sarcoidosis, cancer, multiple sclerosis, amyotrophic lateral sclerosis, Guillain-Barre syndrome, autism, pediatric autoimmune neuropsychiatric disorders, Alzheimer's disease, Tourette's syndrome, diabetes, obesity, cardiovascular disease, polycystic kidney disease, myasthenia gravis and several skin conditions. Infections are one of the most common preventable causes of cancer [50].

\section{Autoreactivity in chronic urticaria}

Nearly one third of cases of spontaneous chronic urticaria is caused by autoreactivity (30\% of the patients have positive autologous serum skin test). Autoimmunity occurs when the immune system of the host recognizes and attacks its own tissues. There are many factors responsible for the autoimmune response, such as genetics, age and environment. Environmental factors triggering the development of the immune system response include bacterial and viral infections [51]. In chronic urticaria there is production of IgG antithyroid antibodies and histamine releasing autoantibodies to the FceRI (high-affinity lgE receptor) or to IgE $[52,53]$. The associa- tion of HLA class II alleles, such as HLA-B44, DQB1*0302, HLA-DRB1*01, HLA DRB1*04, HLA-DRB*15, confirms the autoimmune theory of the pathogenesis of chronic urticaria and may be the genetic factor in this process [5456]. Serum of the patients with the positive result of the autologous serum skin test had significantly increased histamine release compared with skin test-negative chronic urticaria sera, as well as higher induction of basophile CD63 surface expression and sulfidoleukotriene production. Additionally, incubation with chloroquine, dapsone, and lidocaine caused reduction in the histamine release, CD63 expression, and sulfidoleukotriene production [57].

As a part of the Focal Infection Theory, host infection by the pathogen (virus, bacteria) leads to autoimmunity. If the pathogen carries amino acids similar to the self-antigen, lymphocytes $T$ or $B$ are activated due to cross-reaction and lead to the damage and activation of the other parts of the immune system. This mechanism is called 'molecular mimicry'. Persisting pathogens stimulate the immune response and the lysis process, which leads to release in antigens. Antigens from the damaged tissues interact with antigen-presenting cells (APC) and cause selfspecific immune response. In the mechanism of 'bystander activation', the inflammatory environment of the infection activates one arm of the immune system, leading to the domino effect with subsequent activation of the other paths. The inflammatory process can also lead to processing and presentation of 'cryptic antigens' normally invisible to the immune system [57]. Several pathogens have been described in the chronic urticaria, but only in Helicobacter pylori, streptococcal, staphylococcal and Yersinia infections those mechanisms have been proved [58, 59]. A streptococcal infection is an example of molecular mimicry between hemolytic streptococcus group A antigens and proteins of the host. This leads to both humoral and cell-mediated autoimmune reactions and clinical consequences of rheumatic fever and heart disease [60]. The majority of the studies emphasize the role of Helicobacter pylori as the exacerbating factor of the chronic urticaria. The prevalence of Helicobacter pylori is higher when an additional thyroid autoimmunity process occurs [61]. Eradication of Helicobacter pylori causes often remission of chronic urticaria. Studies show that the remission rate is $61.5 \%$ in patients with eradicated Helicobacter pylori and $33.6 \%$ in the non-treated group. In $39 \%$ of patients persistent yersiniosis was observed. Treatment with quinolones led in some cases to remission of urticaria [62]. Norovirus is a viral cause of urticaria [63]. The parasitic factors in chronic urticaria include Blastocystis hominis, Giardia lamblia, Trichinella, Trichomonas vaginalis and Toxocara canis (35-46\% of patients) [64-66].

\section{Infections of oropharynx}

Several studies show an increased incidence of tonsillitis, sinusitis and dental infections among patients with 
chronic urticaria. Wedi et al. found sinusitis in $32 \%$ of cases of chronic urticaria and dental infections in $29 \%$ of the patients [67]. Buss et al. found tonsillitis and sinusitis in $50 \%$ of patients [68]. In 10-42\% of analyzed patients with chronic urticaria, anti-streptococcal antibodies were found. Ten percent of the patients had anti staphylolysin antibodies [57]. Buchter et al. and Goga et al. did not find a significant increase of oropharyngeal infections (tonsillitis, sinusitis, caries, periodontitis, periapical lesions, and nonvital and missing teeth) in patients with chronic urticaria. There have been single cases of the recurrence of chronic urticaria after antibiotic treatment [57].

\section{Aim}

There are discordant opinions about the role of focal infections in the pathogenesis of psoriasis and urticaria. The aim of the study was to establish whether there is a higher incidence of focal infections in patients with chronic urticaria and psoriasis.

\section{Material and methods}

We retrospectively reviewed 129 patients with a history of psoriasis and chronic urticaria: 58 women and 71 men treated in the Department of Dermatology of the Jagiellonian University Medical College in Krakow. We collected the following data: patients' age, sex, diagnosis (type of psoriasis, urticaria), aggravating factors, type of consultation (gynecological, dental, laryngological) with its result and C-reactive protein (CRP) value (as the marker of the inflammatory process). We examined 101 patients with psoriasis: 77 patients had plaque psoriasis, 16 had psoriatic arthritis, 4 presented with pustular psoriasis, 4 with psoriatic erythroderma and 28 patients had chronic urticaria. The median age of the patients was 47.91 years with the youngest aged 19 and the oldest 87.

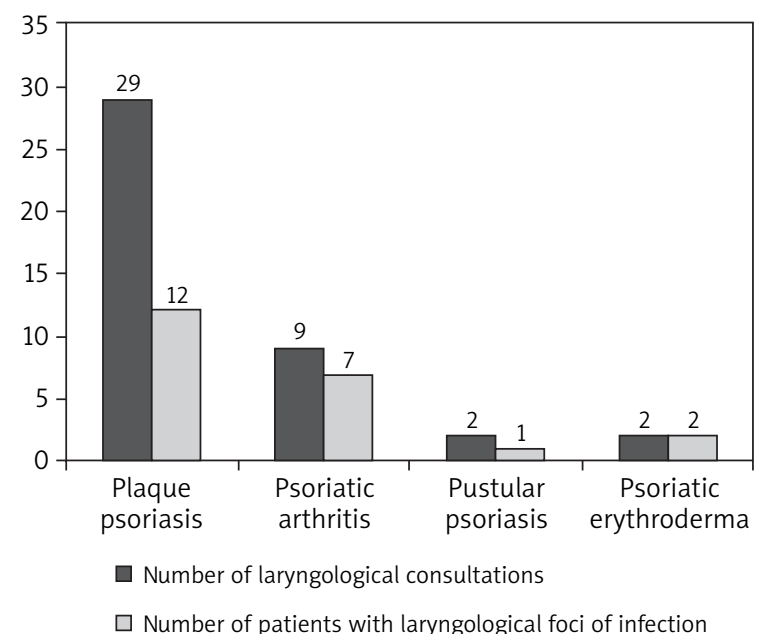

Figure 1. Focal infections (laryngological) in patients with psoriasis

\section{Results}

In the analyzed group, 11 patients had a dental consultation, 58 - laryngological consultation and 29 women had a gynecological consultation.

\section{Psoriasis (men and women)}

The medium age of patients with psoriasis was 48.49 years (19-87 years). Seventy-seven patients had plaque psoriasis, 16 presented with psoriatic arthritis, 4 with pustular psoriasis and 4 with psoriatic erythroderma. In different types of psoriasis, the percentage of women and men was as follows: - plaque psoriasis: 23 women (29.87\%), 54 men (70.13\%), - psoriatic arthritis: 7 women (43.75\%), 9 men (56.25\%), - pustular psoriasis: 3 women (75\%), 1 man (5\%),

- psoriatic erythroderma: 1 woman (25\%), 3 men (75\%) (Figure 1).

Forty-two patients had a consultation with a laryngologist (29 with plaque psoriasis, 9 with psoriatic arthritis, 2 with pustular psoriasis and 2 with psoriatic erythroderma), 9 patients had a dental consultation and 16 women were consulted by a gynecologist.

In the studied group, 24 patients had a laryngological disorder. Among patients with plaque psoriasis 12 had a laryngological focus of infection $(41.38 \%$ of the patients with plaque psoriasis). A laryngological source of infection was found in 7 patients with psoriatic arthritis (43.75\%), 1 with pustular psoriasis (25\%) and 2 with psoriatic erythroderma (50\%). Eight patients (7.92\%) had a dental focus of infection.

\section{Women with psoriasis}

Among 34 women with psoriasis, 23 had plaque psoriasis (68\% of women), 7 had psoriatic arthritis, (20\%), 3 women had pustule psoriasis (9\%) and 1 woman had psoriatic erythroderma (3\%) (Figure 2).

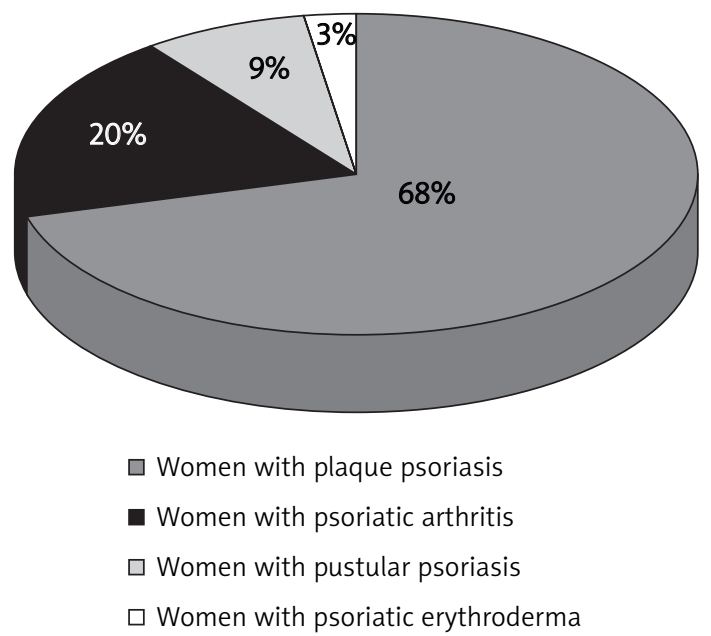

Figure 2. Women with psoriasis 


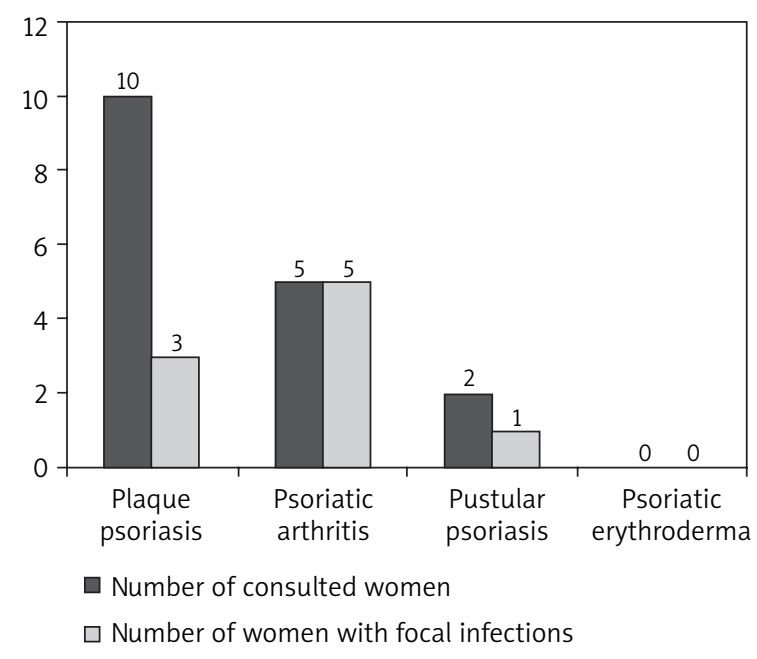

Figure 3. Foci of infection (laryngological) in women with different types of psoriasis

In the group of women with psoriasis, 16 patients had a laryngological consultation, 16 women had a gynecological consultation and 3 - a dental consultation. Ten women had laryngological diagnosis (29.41\% of women), 4 women had gynecological diagnosis (11.76\%) and 3 had dental diagnosis (8.82\%). Thirteen women had at least one focus of infection (38.24\% of women). Four women had 2 foci of infection. The numbers of patients with laryngological and gynecological consultations are shown in Figures 3 and 4.

\section{Men with psoriasis}

Among men with psoriasis, median age was 47.99 years old (19-86) (Figure 5). Fifty-four men (81\%) had plaque psoriasis, 9 men had psoriatic arthritis (13\%), 1 man had pustular psoriasis (2\%) and 3 patients had psoriatic erythro-

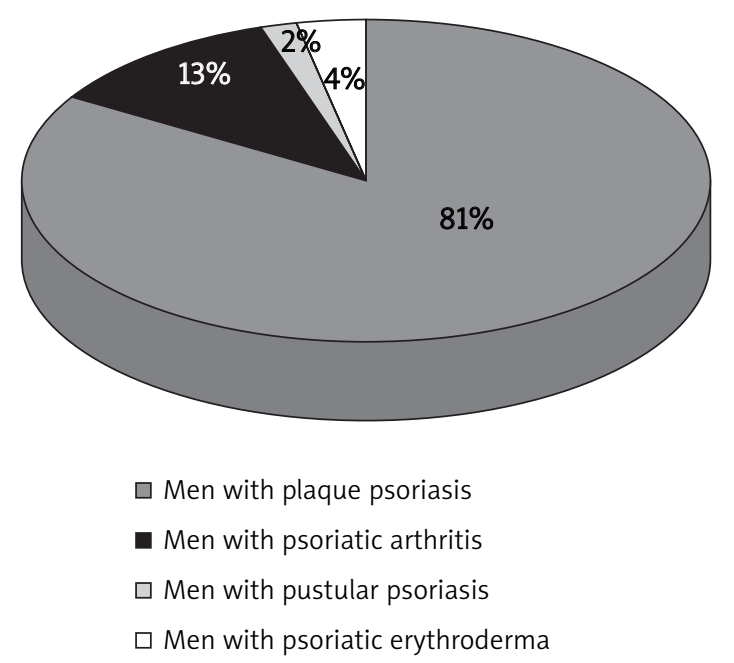

Figure 5. Men with psoriasis



$\square$ Number of women examined (gynecological consultation)

$\square$ Number of women with gynecological diagnosis

Figure 4. Foci of infection (gynecological) in women with different types of psoriasis

derma (4\%). Twenty-five patients were consulted laryngologically and 6 had a dental consultation. Five patients had a dental inflammatory process (4 patients with plaque psoriasis and 1 patient with psoriatic arthritis). Four patients (5.97\% of male patients with psoriasis) had a dental focus of infection, 13 patients had laryngological diagnosis (19.4\%). Nineteen male patients with psoriasis (28.36\%) had a focus of infection localized in the orophar$y n x$. None of the patients had more than one focus of infection (Figure 6).

\section{Patients with urticaria (men and women)}

We examined 28 patients with urticaria, 4 men and 24 women. The medium age of the analyzed group was 45.86 (patients aged: 22-77). Sixteen patients were consulted by

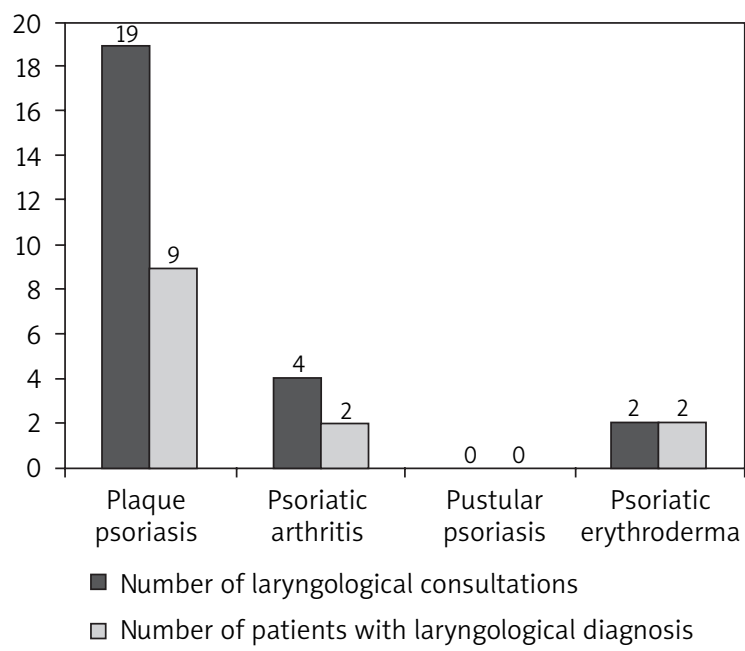

Figure 6. Focal infections (laryngological) in men with psoriasis 
a laryngologist, 13 women by a gynecologist, and 3 patients by a dentist. Three patients (10.71\%) had a focus of infection localized in the dental area, 8 patients $(28.57 \%)$ - in sinuses and the respiratory tract (laryngological diagnosis), 1 woman had a focus of infection localized in the urogenital tract (3.57\% of all patients). Among them, 2 patients had 2 foci of infection. Ten of 28 patients with urticaria (35.71\%) had at least one type of focus of infection. All the cases of focal infections among patients with urticaria appeared in women.

\section{C-reactive protein values}

Medium CRP value in the patients with psoriasis was $12,714 \mathrm{mg} / \mathrm{l}$ and $5.4 \mathrm{mg} / \mathrm{l}$ in the patients with hives.

\section{Aggravating factors in psoriasis and urticaria}

Among patients with urticaria, 11 patients (39.29\%) reported factors aggravating the symptoms of urticaria. The worsening of the clinical status of patients was observed after exposure to drugs (3 patients), particular food (3 patients), stress (3 patients), physical factors (1 patient) and plants (1 patient). Among patients with psoriasis, the influence of the exogenous factor was observed in 17 patients (16.83\%). Two patients reported aggravation of skin changes after drug administration, one after particular food, 5 after exposure to stressful situations, 5 caused by physical factors, 4 reported other factors, but could not determine them.

\section{Types of focal infections}

In patients with urticaria, the most common laryngological sources of infection were sinusitis with nasal septum deformity (8 patients), upper respiratory tract infection (3) and tonsillitis (2). Among gynecological causes, 2 changes were localized in the uterus, 2 - in the cervix, one in the vagina (bacterial vaginosis). One patient had a deep carious lesion. Among patients with psoriasis, 10 had tonsillitis, 9 - upper respiratory tract infection, 5 - sinusitis with nasal septum defect, 2 - nasal polyps and 2 - otitis. Dental foci of infection were deep carious lesions (10). Gynecological sources of infection were cervical (3), vaginal (1) and ovarian (1).

\section{Discussion}

The analysis of data obtained from the patients' documentation provides statistical information on the incidence of psoriasis and urticaria among adults in the region of Krakow. The most common type of psoriasis is plaque psoriasis, followed by psoriatic arthritis, pustular psoriasis and rare psoriatic erythroderma. The majority of the patients with psoriasis were male, with $70.13 \%$ of male patients with plaque psoriasis, $56.25 \%$ with psoriatic arthritis, $25 \%$ with pustular psoriasis and $75 \%$ with psoriatic erythroderma. There was a significantly higher incidence of plaque psoriasis in men (81\%) than in women (68\%).
The incidence of focal infection was higher in women (38.24\%) vs. $28.36 \%$ in men. A lower statistical incidence of infections may be caused by additional gynecological examinations in women. In this retrospective study, the urological consultation was not included. We assume that the percentage of focal infections would be higher in men. if the patients were consulted by an urologist. Among patients with urticaria, $35.71 \%$ of patients had a focus of infection, which confirms results presented in the previous studies concerning this topic. Wedi et al. found sinusitis in $32 \%$ of cases of chronic urticaria and $29 \%$ of dental infection cases [67]. In 10-42\% of patients with chronic urticaria, anti-streptococcal antibodies were found [68]. Patients with psoriasis had a higher CRP level (result above the reference range) in comparison to patients with urticaria (result within the reference range). The most common examples of focal infection were tonsillitis, upper respiratory tract infections, sinusitis, dental caries and genitourinary tract infections. Aggravating factors were similar to previously described ones.

\section{Conclusions}

A high incidence of focal infections in patients with psoriasis and urticaria suggests that infections may play a significant role in pathogenesis of these skin disorders. Treatment of infection foci may play a key role in the remission of skin changes.

\section{References}

1. Sheikh J. Autoantibodies to the high-affinity IgE receptor in chronic urticaria: how important are they? Curr Opin Allergy Clin Immunol 2005; 5: 403-7.

2. Kulthanan K, Jiamton S, Thumpimukvatana N, Pinkaew S Chronic idiopathic urticaria: prevalence and clinical course. J Dermatol 2007; 34: 294-301.

3. Śpiewak R. The substantial differences between photoallergic and phototoxic reactions. Ann Agric Environ Med 2012; 19: 888-9.

4. Osmola-Mańkowska A, Silny W, Dańczak-Pazdrowska A, et al. The sun - our friend or foe? Ann Agric Environ Med 2012; 19: 805-9.

5. Krupa Shankar DS, Ramnane M, Rajouria EA. Etiological approach to chronic urticaria. Indian J Dermatol 2010; 55: 33-8.

6. Sabroe RA, Grattan CE, Francis DM, et al. The autologous serum skin test: screening test for auto antibodies in chronic idiopathic urticaria. Br J Dermatol 1999; 140: 446-52.

7. Żukiewicz-Sobczak W, Krasowska E, Zwoliński J, et al. Allergic diseases - current state of knowledge. Postep Derm Alergol 2012; 29: 451-5.

8. Najib U, Bajwa ZH, Ostro MG, Sheikh J. A retrospective review of clinical presentation, thyroid autoimmunity, laboratory characteristics, and therapies used in patients with chronic idiopathic urticaria. Ann Allergy Asthma Immunol 2009; 103: 496-501.

9. Kanani A, Schellenberg R, Warrington R. Urticaria and angioedema. Allergy Asthma Clin Immunol 2011; 7 (Suppl 1): S9. 
10. Jirapongsananuruk O, Pongpreuksa S, Sangacharoenkit P, et al. Identification of the etiologies of chronic urticaria in children: a prospective study of 94 patients. Pediatr Allergy Immunol 2010; 21: 508-14.

11. Yang HY, Sun CC, Wu YC, Wang JD. Stress, insomnia, and chronic idiopathic urticaria: a case-control study. J Formos Med Assoc 2005; 104: 254-63.

12. Confino-Cohen R, Chodick G, Shalev V, et al. Chronic urticaria and autoimmunity: associations found in a large population study. J Allergy Clin Immunol 2012; 129: 1307-13.

13. Kaplan AP, Finn A. Autoimmunity and the etiology of chronic urticaria. Can J Allergy Clin Immunol 1999; 4: 286-92.

14. Kikuchi Y, Fann T, Kaplan AP. Antithyroid antibodies in chronic urticaria and angioedema. J Allergy Clin Immunol 2003; 112: 218.

15. Dreskin SC, Andrews KY. The thyroid and urticaria. Curr Opin Allergy Clin Immunol 2005; 5: 408-12.

16. Brodell LA, Beck LA. Differential diagnosis of chronic urticaria. Ann Allergy Asthma Immunol 2008; 100: 181-8.

17. Buss YA, Garrelfs UC, Sticherling M. Chronic urticaria which clinical parameters are pathogenetically relevant? A retrospective investigation of patients. I Dtsch Dermatol Ges 2007; 5: 22-9.

18. Asero R, Tedeschi A, Riboldi P, Cugno M. Plasma of patients with chronic urticaria shows signs of thrombin generation, and its intradermal injection causes wheal-and-flare reactions much more frequently than autologous serum. J Allergy Clin Immunol 2006; 117: 1113-7.

19. Asero R, Tedeschi A, Coppola R, et al. Activation of the tissue factor pathway of blood coagulation in patients with chronic urticaria. J Allergy Clin Immunol 2007; 119: 705-10.

20. Takahagi S, Mihara S, Iwamoto K, et al. Coagulation/fibrinolysis and inflammation markers are associated with disease activity in patients with chronic urticaria. Allergy 2010; 65: 649-56.

21. Horn MP, Pachlopnik JM, Vogel M, et al. Conditional autoim munity mediated by human natural anti-Fc(epsilon)Rlalpha autoantibodies? FASEB J 2001; 15: 2268-74.

22. Vasagar K, Vonakis BM, Gober LM, et al. Evidence of in vivo basophil activation in chronic idiopathic urticaria. Clin Exp Allergy 2006; 36: 770-6.

23. Jacques $P$, Lavoie A, Bédard PM, et al. Chronic idiopathic urticaria: profiles of skin mast cell histamine release during active disease and remission. J Allergy Clin Immunol 1992; 89: 1139-43.

24. Nevitt GJ, Hutchinson PE Psoriasis in the community: prevalence, severity and patients' beliefs and attitudes towards the disease. Br J Dermatol 1996; 135: 533-7.

25. Gelfand JM, Stern RS, Nijsten T, et al. The prevalence of psoriasis in African Americans: results from a population-based study. J Am Acad Dermatol 2005; 52: 23-6.

26. Rapp SR, Feldman SR, Exum ML, et al. Psoriasis causes as much disability as other major medical diseases. J Am Acad Dermatol 1999; 41: 401-24.

27. Gudjonsson JE, Thorarinsson AM, Sigurgeirsson B, et al. Streptococcal throat infections and exacerbation of chronic plaque psoriasis: a prospective study. Br J Dermatol 2003; 149: 530-4.

28. Fortes C, Mastroeni S, Leffondré K, et al. Relationship between smoking and the clinical severity of psoriasis. Arch Dermatol 2005; 141: 1580-4.

29. Rasmy H, Mikhael N, Ismail S. Interleukin-18 expression and the response to treatment in patients with psoriasis. Arch Med Sci 2011; 7: 713-9.
30. Huerta C, Rivero E, Rodríguez LA. Incidence and risk factors for psoriasis in the general population. Arch Dermatol 2007; 143: 1559-65.

31. Herron MD, Hinckley M, Hoffman MS, et al. Impact of obesity and smoking on psoriasis presentation and management. Arch Dermatol 2005; 141: 1527-34.

32. Balato N, Di Costanzo L, Balato A. Differential diagnosis of psoriasis. J Rheumatol Suppl 2009; 83: 24-5.

33. Krueger JG, Bowcock A. Psoriasis pathophysiology: current concepts of pathogenesis. Ann Rheum Dis 2005; 64 (Suppl II): ii30-6.

34. Rajeev Das, Arun Jain, V Ramesh V. Current concepts in the pathogenesis of psoriasis. Indian J Dermatol 2009; 54: 1, 7-12.

35. Cepicka W, Tielsch R. Focal infections and psoriasis vulgaris. Dermatol Wochenschr 1967; 153: 193-9.

36. Blok S, Vissers WH, Van Duinhoven M, Van de Kerkhof PC. Aggravation of psoriasis by infections: a constitutional trait or a variable expression? Eur J Dermatol 2004; 14: 259-61.

37. Nomura K, Mizutani H, Inachi S, Shimizu M. Remission of pustular psoriasis after cholecystectomy: role of focal infection in pustularization of psoriasis. J Dermatol 1995; 22: 122-4.

38. Mizutani $\mathrm{H}$, Ohmoto $\mathrm{Y}$, Mizutani T, et al. Role of increased production of monocytes TNF-alpha, IL-1beta and IL-6 in psoriasis: relation to focal infection, disease activity and responses to treatments. Dermatol Sci 1997; 14: 145-53.

39. Yamamoto T. Triggering role of focal infection in the induction of extra-palmoplantar lesions and pustulotic arthroosteitis associated with palmoplantar pustulosis. Adv Otorhinolaryngol 2011; 72: 89-92.

40. Krstić A, Zivković M, Janjatović T. Significance of focal infection in psoriasis vulgaris and psoriasis arthropathica. Hautarzt 1969; 20: 274-6.

41. Fujihara K. A study on the tonsil with focal infections-with special reference to the newly devised tonsillar cryptscope and the architecture of vessels in crypts. Nihon Jibiinkoka Gakkai Kaiho 1991; 94: 1304-14.

42. Ruszczak Z, Bednarowicz G, Prószyńska-Kuczyńska W. The role of focal infection in the etiopathogenesis of psoriasis. Przegl Dermatol 1969; 56: 15-20.

43. Fry L, Baker BS. Triggering psoriasis: the role of infections and medications. Clin Dermatol 2007; 25: 606-15.

44. Francke OC. William Hunter's "oral sepsis" as a cause of disease. Periodontology 2000; 23: 13-8.

45. Miller WD. The micro-organisms of the human mouth: the local and general diseases which are caused by them. S.S. White, Philadelphia 1880.

46. Miller WD. The human mouth as a focus of infection. Dent Cosmos 1891; 33: 689-713.

47. Billings F. Chronic focal infections and their etiologic relations to arthritis and nephritis. Arch Int Med 1912; 9: 484-98.

48. Billings F. Focal infection: the lane medical lectures. D. Appleton and Company, New York 1916.

49. Ercolini AM, Miller SD. The role of infections in autoimmune disease. Clin Exp Immunol 2009; 155: 1-15.

50. Boguniewicz M. The autoimmune nature of chronic urticaria. Allergy Asthma Proc 2008; 29: 433-8.

51. Greaves MW, Tan KT. Chronic urticaria: recent advances. Clin Rev Allergy Immunol 2007; 33: 134-43.

52. Coban M, Erdem T, Ozdemir S, et al. HLA class I and class II genotyping in patients with chronic urticaria. Int Arch Allergy Immunol 2008; 147: 135-9.

53. O'Donell BF, O'Neill CM, Francis DM, et al. Human leukocyte antigen class II associations in chronic idiopathic urticaria. Br J Dermatol 1999; 140: 853-8. 
54. Aydogan K, Karadogan SK, Akdag I, Tunali S. HLA class I and class II antigens in Turkish patients with chronic ordinary urticaria. Clin Exp Dermatol 2006; 31: 424-9.

55. Wedi B, Novacovic V, Koerner M, Kapp A. Chronic urticaria serum induces histamine release, leukotriene production, and basophil CD63 surface expression: inhibitory effects of anti-inflammatory drugs. J Allergy Clin Immunol 2000; 105: 552-60.

56. Wedi B, Raap U, Kapp A. Chronic urticaria and infections. Curr Opin Allergy Clin Immunol 2004; 4: 387-96.

57. Wedi B, Liekenbröcker T, Kapp A. Persistent bacterial infec tions and serum acitivity in chronic urticaria - role of molecular mimicry? Allergologie 2001; 24: 480-90.

58. Bakos N, Hillander M. Comparison of chronic autoimmune urticaria with chronic idiopathic urticaria. Int I Dermatol 2003; 42: 613-5.

59. Wedi B, Raap U, Wieczorek D, Kapp A. Urticaria and infections. Allergy Asthma Clin Immunol 2009; 5: 10.

60. Wedi B. Urticaria. J Deutsch Dermatol Ges 2008; 6: 306-17.

61. Leiste A, Skaletz-Rorowski A, Venten I, et al. Urticaria associated with Norovirus infection: report of two cases. J Dtsch Dermatol Ges 2008; 6: 563-23.

62. Trachsel C, Pichler WJ, Helbling A. Importance of laboratory investigations and trigger factors in chronic urticaria. Schweiz Med Wochenschr 1999; 129: 1271-9.

63. Giacometti A, Cirioni O, Antonicelli L, et al. Prevalence of intestinal parasites among individuals with allergic skin diseases. J Parasitol 2003; 89: 490-2.

64. Ronellenfitsch U, Bircher A, Hatz C, Blum J. Parasites as a cause of urticaria. Helminths and protozoa as triggers of hives? Hautarzt 2007; 58: 133-4, 136-41.

65. Wedi B, Kapp A. Urticaria and angioedema. In: Allergy: practical diagnosis and management. Mahmoudi M (ed.). McGraw Hill, New York 2008; 84-94.

66. Buss YA, Garrelfs UC, Sticherling M. Chronic urticaria: which clinical parameters are pathogenetically relevant? A retrospective investigation of 339 patients. J Dtsch Dermatol Ges 2007; $5: 22-7$.

67. Goga D, Vaillant L, Pandraud L, et al. The elimination of dental and sinusal infectious foci in dermatologic pathology. A double-blind study in 27 cases confined to chronic urticaria. Rev Stomatol Chir Maxillofac 1988; 89: 273-5.

68. Buchter A, Kruse-Losler B, Joos U, Kleinheinz J. Odontogenic foci: possible etiology of urticaria? Mund Kiefer Gesichtschir 2003; 7: 335-8. 